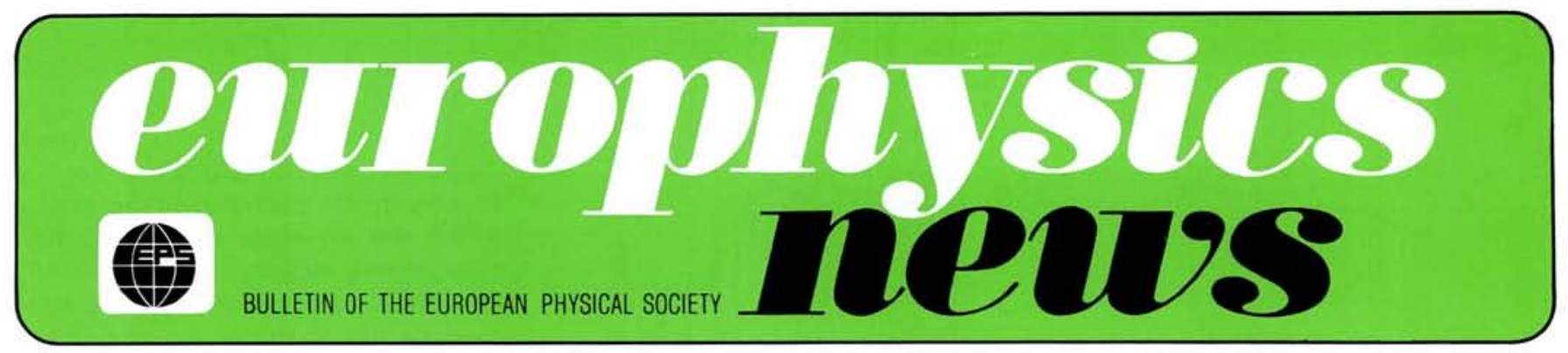

J.A. Volume 19

\title{
The Aharonov-Bohm Effect in Normal Metals
}

\section{Van Haesendonck and Y. Bruynseraede, Leuven}

\author{
(Laboratorium voor Vaste Stof-Fysika en Magnetisme, Katholieke Universiteit)
}

In 1924, de Broglie introduced his famous quantum-mechanical wave picture to describe the motion of electrons. Freely moving electrons are described as plane waves $\Psi(\vec{r}) \sim \exp (\overrightarrow{i k} \cdot \vec{r})$ and their momentum $p$ varies inversely proportional to the wavelength $\lambda$ :

$$
p=h / \lambda=\hbar|\vec{k}| \text {. }
$$

The validity of the wave picture can be tested directly by studying the diffraction of an electron beam by a periodic array of atoms. In 1927, Davisson and Germer showed that after penetration through a crystal, the diffraction patterns for an electron beam and an X-ray beam are identical.

With the development of the transmission electron microscope, it became also possible to show that the phase of an electron wave has a physical significance. When the electron beam in an electron microscope is split up into two parts using an electrostatic biprism, interference fringes will be formed near the point where the two beams converge. Holographic imaging of an object is also possible if this object is placed in one of the interfering beams.

When a magnetic field $\vec{B}$ is applied, two effects are observed. First of all, the envelope of the interference pattern is shifted because of the Lorentz force $\vec{F}_{L}=-e \vec{v} \times \vec{B}$ which modifies the path followed by the electrons. At the same time, the position of the interference fringes shifts relative to the envelope of the pattern. This shift is usually referred to as the Aharonov-Bohm effect. As first predicted by Aharonov and Bohm in 1959 [1], the phase shift between the interfering beams only depends upon the total magnetic flux $\Phi_{B}$ enclosed by the interfering beams. Careful experiments confirmed that the AharonovBohm effect occurs even when the flux $\Phi_{B}$ is completely confined to the interior of the electron path so that $F_{\mathrm{L}}=0$.

It is important to note that the Aharonov-Bohm effect is a direct consequence of the wave character of the electrons and does not have a classical analogue. In order to understand this non-local influence of the magnetic flux, one has to assume that the vector potential $\vec{A}$ influences the electron waves even in regions where $\vec{B}=\nabla \times \vec{A}=0$. The Aharonov-Bohm effect relies on the fact that the phase of the wave function which is calculated from the Schrödinger equation, depends upon the specific choice for the vector potential. Contrary to the physical observable quantities, the phase of a wave function is not gauge invariant.

As pointed out by Aharonov and Bohm, the wave function of an electron which moves in a region where $\vec{A} \neq 0$, will pick up an extra phase $\varphi$ which is determined by the line integral of the vector potential along the electron path:

$$
\varphi=(e / \hbar) \int \vec{A} \cdot d \vec{l} \text {. }
$$

For a closed path, Eq. (2) predicts a modulation of the phase by the enclosed magnetic flux:

$$
\begin{aligned}
\varphi=(e / \hbar) \oint \vec{A} \cdot d \vec{l} & =(e / \hbar) \iint \vec{B} \cdot d \vec{S} \\
& =2 \pi \Phi_{B} / \Phi_{0}
\end{aligned}
$$

where the flux quantum $\Phi_{0}=h / e$. Equation (3) has been successfully used to explain the magnetic-flux quantization within a hollow superconducting cylin- der. In a superconductor the electrons form Cooper pairs with charge $2 e$. The enclosed magnetic flux will be an integer multiple of the superconducting flux quantum $h / 2 e=\Phi_{\mathrm{o}} / 2$.

Since 1966, the fundamental question has been raised whether the Aharonov-Bohm effect can also be observed for the conduction electron waves in a normal metal [2]. At high temperatures, the inelastic scattering of the conduction electrons (by other electrons or by phonons) will cause random variations of the electronic wavelength on an atomic length scale and therefore destroy the interference between conduction electron waves. At low temperatures, the phase coherence length $L_{\varphi^{\prime}}$ i.e. the length over which the electrons can diffuse elastically without changing their wavelength, can not exceed the

\section{Contents}

The Aharonov-Bohm Effect in Normal Metals

Surface Crystallography by X-ray Diffraction

New Members of EPS

The European Very Large Telescope Project

EPS Workshops The Future of MagnetoOptical Recording SOUID - State of the Art

History of Physics

Latest SUN Revision

Meetings Up-Date

Commercial Plasma Exhibit 


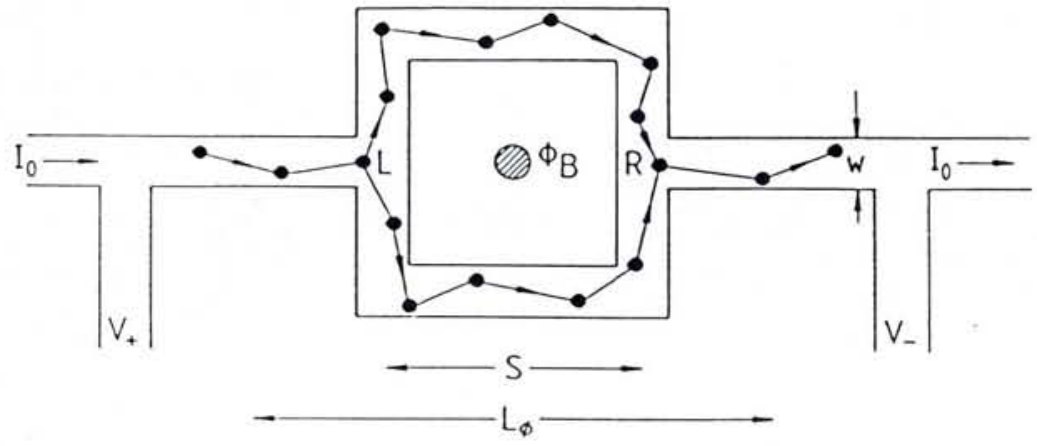

Fig. 1 - Four-terminal measurement of the interference between split conduction electron waves in a disordered metal film loop. The black dots represent the elastic scattering of the electron waves at lattice defects or impurities. As predicted by Aharonov and Bohm, an interference pattern with flux period $\Phi_{O}=h / e$ is generated by the flux $\Phi_{B}$ which is completely confined to the interior of the loop. When the two partial waves travel completely around the loop and return to point $L$, the flux period for the magnetoresistance oscillations will be the superconducting flux quantum $\mathrm{h} / 2 \mathrm{2}$.

inelastic diffusion length $L_{\text {in }}=\left(D \tau_{\text {in }}\right)^{1 / 2}$, where $D$ is the diffusion constant.

Typical quantum effects such as the Shubnikov - de Haas - Van Alphen magnetoresistance oscillations or the quantum Hall effect, can only be observed in very pure, high mobility samples. One is therefore tempted to believe that elastic disorder scattering destroys the quantum coherence. Here we shall show that the quantum coherence between interfering electron waves survives in the presence of the elastic defect scattering. The experiments we discuss in the next sections, prove that elastic scattering even enhances the influence of the interference effects upon the electronic conduction. From a theoretical point of view, much insight has been gained from the Landauer description [3], which treats the disorder as a tunnelling barrier for the conduction electrons. In an isolated ring of a normal metal (no leads attached), the cohecurrent, comparable to a superconducting current, can be induced by applying a time-dependent magnetic flux $\Phi_{B}(t)$. The classical, resistive behaviour only appears when the inelastic (phase breaking) scattering is introduced.

\section{Interference Processes in Normal Metals}

The basic configuration which is needed to observe the solid-state analogue of the Aharonov-Bohm effect is sketched in Fig. 1. A conduction electron wave arriving at point $L$ is divided into two partial waves which diffuse elastically along both arms of the square metal loop of size $\mathrm{S}$ and interfere at point R. The phase coherence length $L_{\varphi}$ is comparable to the distance $2 \mathrm{~S}$ between the points $L$ and $R$. The black dots in Fig. 1 represent the elastic scattering events where the $\vec{k}$ vector of the electron changes its direction. The probability $P_{\mathrm{R}}$ that the electron arrives at point $R$, is given by the square of the sum of the two partial wave functions $\Psi_{1}(\mathrm{R})$ and $\Psi_{\| 1}(\mathrm{R})$ which describe the electron in the upper and lower branch of the metal loop:

$$
\begin{gathered}
P_{\mathrm{R}}=\left|\Psi_{1}\right|^{2}+\left|\Psi_{\|}\right|^{2}+\Psi_{1}^{*} \Psi_{\| 1} \exp (i \theta) \\
+\Psi_{\|}^{*} \Psi_{1} \exp (-i \theta) .
\end{gathered}
$$

The wave character of the conduction electrons causes interference terms whose magnitude depends upon the phase shifts $\delta_{1}(i)$ and $\delta_{\|}(j)$ occurring during each of the elastic scattering events. The phase factor $\theta$ is given by:

$$
\theta=\Sigma_{i} \delta_{1}(i)-\Sigma_{j} \delta_{\| 1}(j) \text {. }
$$

Depending upon the impurity configuration in the upper and lower branch, the interference at point $R$ is either constructive or destructive. When a magnetic flux $\Phi_{B}$ is applied, the AharonovBohm effect induces an additional phase difference $\varphi$ given by Eq. (3). Therefore, the probability $P_{R}\left(\Phi_{B}\right)$ will be an oscillating function of $\Phi_{B}$. From Eq. (4) we find that

$$
\begin{aligned}
P_{R}\left(\Phi_{B}\right)= & \left|\Psi_{1}\right|^{2}+\left|\Psi_{\|}\right|^{2}+\Psi_{1}^{*} \Psi_{\|} \exp (i(\varphi+\theta)) \\
& \quad+\Psi_{\|}^{*} \Psi_{1} \exp (-i(\varphi+\theta)) \\
= & 2\left|\Psi_{1}\right|^{2}[1+\cos (\varphi+\theta)] \\
= & 2\left|\Psi_{1}\right|^{2}\left[1+\cos \left(\left\{2 \pi \Phi_{B} / \Phi_{o}\right\}+\theta\right)\right] \quad(6)
\end{aligned}
$$

Fig. 2 - Experimental observation of the AAS magnetoresistance oscillations with flux period $\Phi_{o}=h / 2 e$ in thin-walled $M g$ cylinders. The cylinders are fabricated by evaporating a thin $\mathrm{Mg}$ film (thickness $d \cong 10 \mathrm{~nm}$ ) onto a very fine quartz fibre which has been stretched over a hole in a rotating glass substrate. The magnetoresistance curves have been obtained for three cylinders with different diameter $\phi=2 r$. Since the coherent back-scattering will be destroyed when $L_{\varphi}<\phi$, the experiments indicate that $L_{\varphi} \cong 2 \mu \mathrm{m}$ at the temperature $T=1.5$ $K$ where the magnetoresistance has been measured. (From ref. 8)
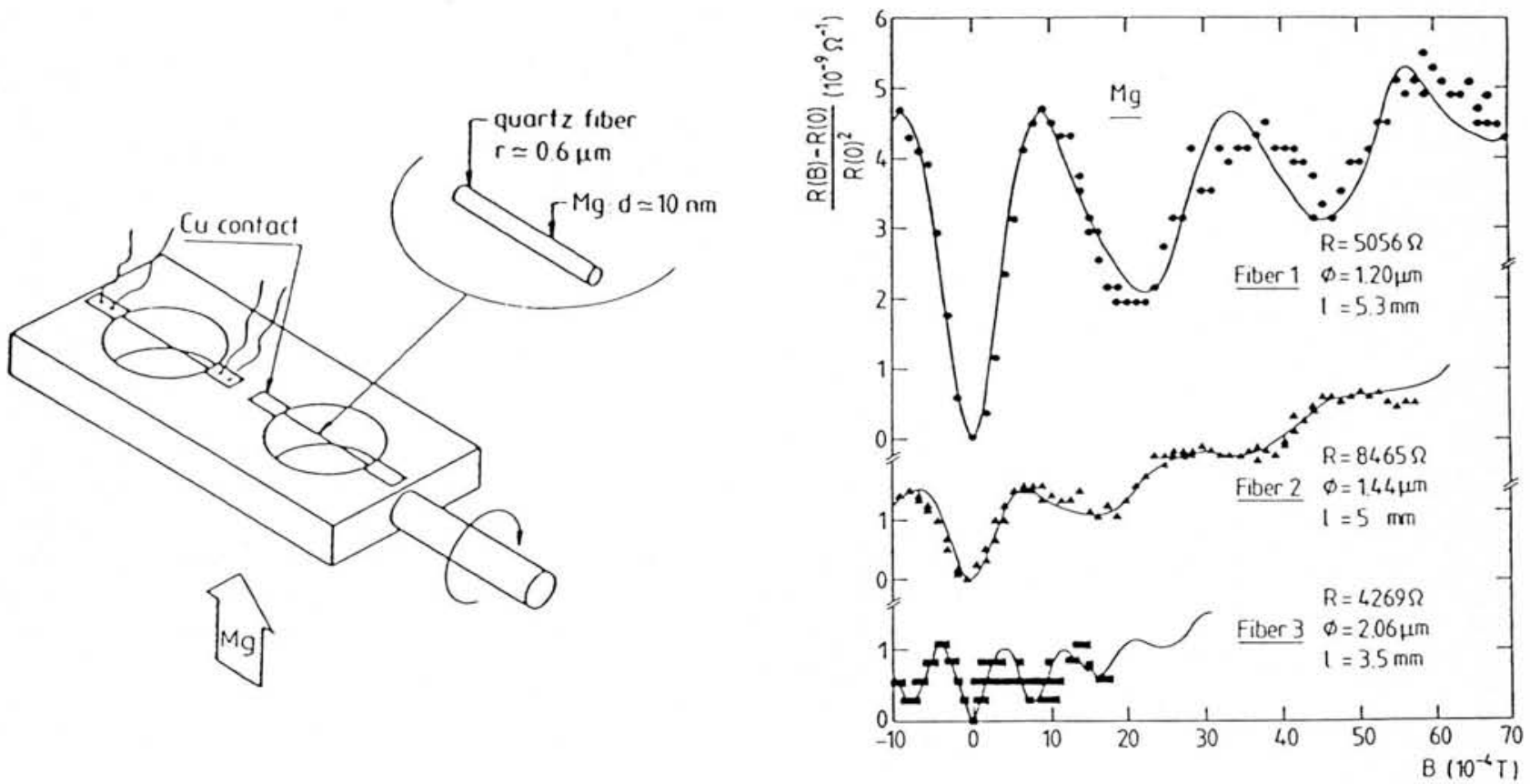
if we assume that in zero magnetic field, the probability to diffuse via the upper and the lower branch is identical $\left(\left|\Psi_{1}(\mathrm{R})\right|^{2}=\left|\Psi_{\|}(\mathrm{R})\right|^{2}\right)$.

Eq. (6) is valid only for a single electron. In a real metal loop, a dense gas of conduction electrons with velocity $v_{F}$ is moving through the arms of the loop. Each of the electrons will diffuse along a different path with a different phase factor $\theta$. Because of the statistical averaging over the different paths, the interference effects will be strongly reduced and the conductivity is proportional to the average elastic mean free path $I_{\text {el }}$ as predicted by the classical transport theories which completely neglect the influence of quantum interference.

Since 1981, theoretical calculations and detailed experiments clearly indicated that the classical Boltzmann theory has to be corrected when one considers the electronic conduction on a length scale which is smaller than the phase coherence length $L_{\varphi}$. Since for pure metals $L_{\varphi} \cong 1 \mu \mathrm{m}$ at very low temperatures, this regime is usually referred to as the "mesoscopic regime". This regime separates the microscopic, atomic regime where quantum mechanics dominate, from the macroscopic regime where classical mechanics are valid. As first suggested by Gefen, Imry and Azbel [4], the conductance $G=I_{0} /\left(V_{+}-V_{-}\right)$ of the mesoscopic loop shown in Fig. 1, will oscillate around its average value $G$ 。 as a function of the magnetic flux $\Phi_{\mathrm{B}}$ threading the loop:

$$
G \cong G_{0}+G_{\text {un }} \cos \left[\left(2 \pi \Phi_{\mathrm{B}} / \Phi_{\mathrm{o}}\right)+\theta\right]
$$

$$
+ \text { higher harmonics }
$$

where the conductance $G_{\text {un }}=e^{2} / h \cong$ $4 \times 10^{-5} \Omega^{-1}[5]$. Owing to the exponential damping of the magneto-conductance oscillations for $2 S>L_{\varphi^{\prime}}$, the amplitude of the higher harmonic oscillations will be small when compared to the amplitude of the $h / e$ oscillations.

Although in Eq. (7), the arbitrary phase factor $\theta$ is still present, the amplitude of the $h / e$ conductance oscillations is independent of the sample size and the amount of disorder as long as $L_{\varphi} \cong$ $2 S$. The conductance oscillations may be considered as statistical fluctuations of the conductance $G$. Equation $(8) \mathrm{im}$ plies that the conductance is not a selfaveraging property in the mesoscopic regime and the conductance fluctuations have a "universal" amplitude comparable to $G_{u n}=e^{2} / h$, independent of the amount of impurity averaging. For a macroscopic sample containing a large number $N$ of mesoscopic units, the amplitude of the interference effects will be reduced by a factor $\sqrt{ } N$.

\section{UNIVERSITÉ DE GENÈVE}

\section{SCHOLA GENEVENSIS MDLIX}

La Faculté des Sciences ouvre une inscription pour un poste de

\section{MAÎTRE D'ENSEIGNEMENT ET DE RECHERCHE}

au Département de Physique théorique.

Charge: II s'agit d'un poste à charge complète, comprenant l'enseignement (env. 2 heures hebdomadaires) et recherche en physique théorique et mathématique (mécanique statistique, dynamique classique et quantique, théorie des champs). Tâches organisatrices.

Titre exigé: Doctorat ou titre jugé équivalent.

Entrée en fonction: 1er octobre 1989 ou date à convenir.

Les dossiers de candidatures doivent être adressés avant le 15 octobre 1988 au Directeur du Département de physique théorique, 24, Quai Ernest Ansermet, CH-1211 Genève 4, auprès duquel des renseignements complémentaires peuvent être obtenus sur le cahier des charges et les conditions.

\section{Aharonov-Bohm Effect in the Macro- scopic Regime}

Since the development in 1979 of the scaling theory for the metal-insulator transition, it has become clear that a special class of interference processes survives the impurity averaging on a macroscopic scale [6]. These interference processes are caused by two partial waves which travel completely around the loop shown in Fig. 1, and interfere again at point $L$. Since the two wave functions $\Psi_{1}(L)$ and $\Psi_{\| 1}(L)$ correspond to time-reversed states, the phase factor $\theta \equiv 0$ in Eq. (6). Consequently, the magneto-conductance oscillations with flux period $\Phi_{0}=h / 2 e$ resulting from this coherent back-scattering on a mesoscopic scale, survive in a macroscopic sample and are directly related to the well-known weak electron localisation in thin metal films.

When a thin-walled metal cylinder with diameter $\phi=2 r$ is placed in an axial magnetic field, all the electron waves that are elastically back-scattered around the cylinder, will enclose the same magnetic flux $\Phi_{B}=\pi r^{2} B$. In 1981, Altshuler, Aharonov and Spivak (AAS) [7] used a Green's function perturbation calculation to show that the resistance $R$ measured between the top and the bottom of the cylinder will oscillate with a flux period $h / 2 e$ and an amplitude $\Delta R$ given by $\Delta R / R \cong$

$$
G_{\text {un }} R_{\square} \cos \left(4 \pi \Phi_{B} / \Phi_{o}\right) K_{o}\left(2 \pi r / L_{\phi}\right) .
$$
$R_{\square}$ is the resistance per square of the metal cylinder wall and $K_{0}(x)$ is the McDonald function. When the cylinder diameter $\phi$ becomes larger than the phase coherence length $L_{\varphi^{\prime}}$ the McDonald function results in an exponential damping of the resistance oscillations. Apparently, the amplitude of the $h / 2 e$ oscillations can be enhanced considerably by increasing the disorder so that the resistance per square $R_{\square}$ becomes comparable to $G_{\text {un }}^{-1}$. Unfortunately, the inelastic diffusion length $L_{\text {in }}$ varies inversely proportional to $R_{\square}$ in disordered metal films. The exponential damping caused by the decrease of the phase coherence length $L_{\varphi}$ will be much stronger than the enhancement of the oscillation amplitude caused by a larger $R$ value. It is important to note that the AAS $h / 2 e$ oscillations do not correspond to the higher harmonic $h / 2 e$ AharonovBohm oscillations given by Eq. (7). Since the AAS oscillations result from the interference between time-reversed states, these oscillations will be destroyed by a high magnetic field or by the presence of magnetic impurities. This effect is very similar to the well-known destruction of Cooper pairs in a superconductor. On the other hand, the higher harmonic Aharonov-Bohm oscillations should still be present at high magnetic fields.

Experimentally, thin-walled metal cylinders with diameter $\phi \cong 1 \mu \mathrm{m}$ can be fabricated by evaporating a $20 \mathrm{~nm}$ thick metal film onto a quartz fibre which is stretched over a hole (diameter $\sim 1 \mathrm{~cm}$ ) in a glass substrate, as shown in Fig. 2 . During the metal evaporation, the substrate is rotated in a reduced helium atmosphere to ensure a uniform thickness of the cylinder wall. In Fig. 2, we also show the low field magnetoresistance (field parallel to the fibre axis) for three Mg cylinders with different diameter $\phi$ at $T \cong 1.5 \mathrm{~K}$. These experimental results, obtained at Leuven in 1983 [8], clearly confirm the existence of the $h / 2 e$ oscillations which were observed for the first time by Sharvin and Sharvin in 1981 [9]. As shown by the full curves in Fig. 2, the $h / 2 e$ oscillations can be nicely fitted by the AAS theory when we assume a phase coherence length $L_{0} \cong 2.0 \mu \mathrm{m}$. The exponential damping of the oscillation amplitude which occurs when $L_{\varphi}<$ $\phi$ is clearly present. The disappearance of the $h / 2 e$ oscillations at higher magnetic fields, is mainly due to the magnetic field penetrating into the cylinder walls. 


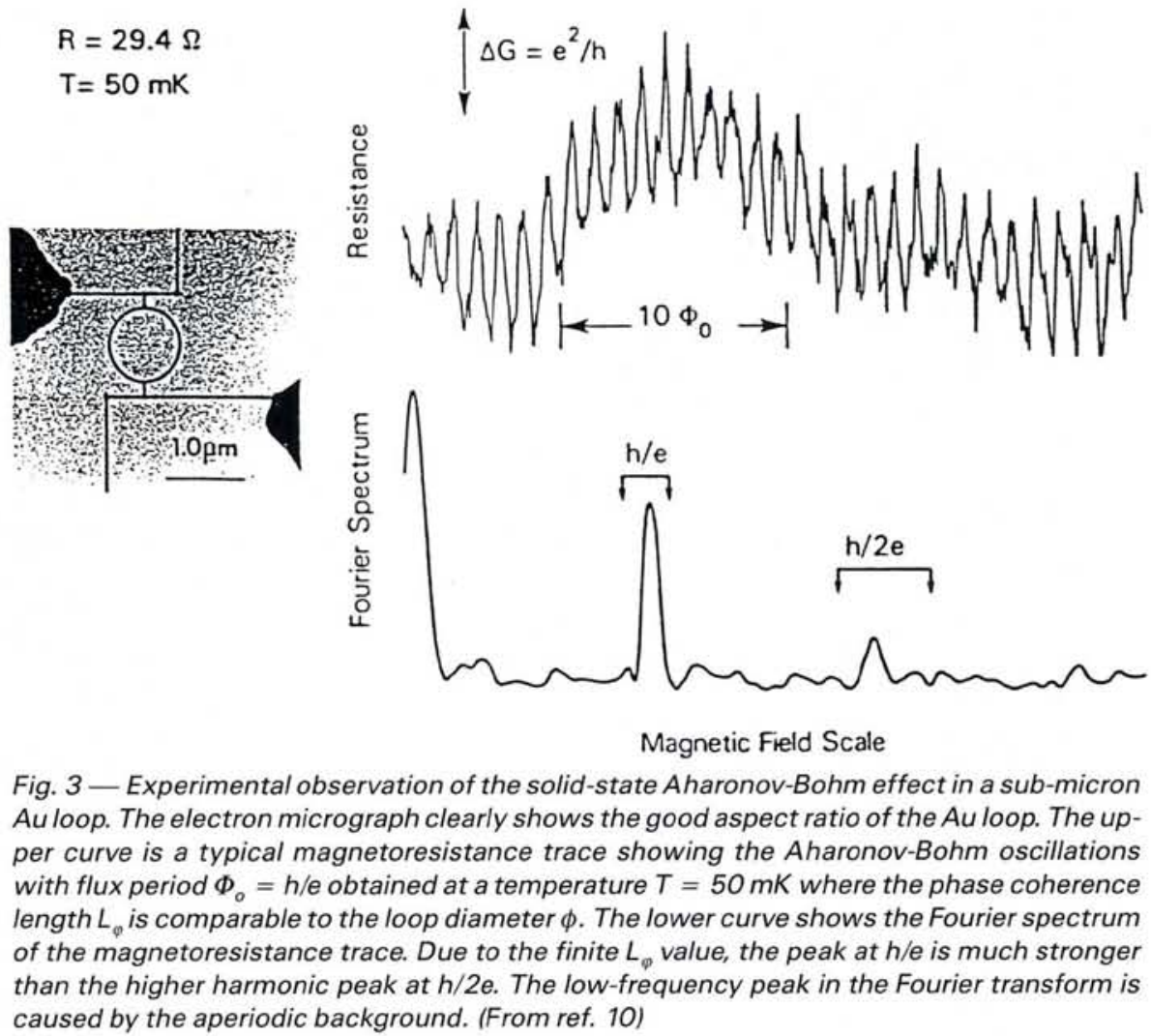

flux penetrating the arms of the loop. Direct interference processes occurring within the arms of the loop will produce sample specific oscillations ("'magneto fingerprints") with different flux periods which depend on the area that is thereby enclosed.

In 1984, Webb and his coworkers [10] succeeded in fabricating a gold ring with a very good aspect ratio. This loop with a diameter $\phi \cong 0.8 \mu \mathrm{m}$ and $w \cong d \cong 0.04$ $\mu \mathrm{m}$ was obtained by "contamination" lithography in a transmission electron microscope. Fig. 3 shows an electron micrograph of this mesoscopic loop together with a typical magnetoresistance trace which clearly shows the $h / e$ oscillations. Because of the good aspect ratio, the periodic $h / e$ oscillations can be easily distinguished from the aperiodic fluctuations which appear as a slowly varying background. Fig. 3 also shows the Fourier transform of the magnetoresistance trace. The sharp peak corresponding to the $h / e$ effect is much stronger than the $h / 2 e$ effect, in agreement with an exponential damping of the higher harmonic effects due to the finite
This penetration causes a destruction of the time-reversal symmetry between the two partial electron waves which are back-scattered around the cylinder in clockwise and counter-clockwise direction.

\section{Aharonov-Bohm Effect in the Mesosco- pic Regime}

The experiments performed on metal cylinders have clearly proved that interference processes between electron waves influence the electronic conduction even on a macroscopic scale. The coherent back-scattering, which causes the weak electron localization in a plane metal film, becomes directly observable via the $h / 2 e$ magnetoresistance oscillations. Equation (7) predicts that the $h / e$ oscillations caused by the direct interference between split electron waves should dominate the $h / 2 e$ oscillations if one would succeed in preparing a mesoscopic loop having a width and a thickness $w, d \ll L_{\varphi}$. Recent developments in electron beam lithography made it possible to prepare mesoscopic loops with a diameter $\phi \leq 1 \mu \mathrm{m}$. The first experiments performed by Webb, Washburn, Umbach and Laibowitz [10] failed to show periodic $h / e$ oscillations. Instead, aperiodic (but reproducible) magnetoresistance fluctuations appeared with an amplitude which strongly increased at lower temperatures. As pointed out by Stone [11], the aperiodic oscillations are caused by the magnetic
Fig. 4 - The slow ensemble averaging of direct interference processes in finite arrays of $\mathrm{Ag}$ loops. The electron micrograph shows a typical sample with $N=3$ units. In the finite arrays, the incoherent addition of the direct interference processes results in a stochastic averaging of the $h / e$ oscillation amplitude inversely proportional to $\sqrt{ } N$. The $h / 2 e$ oscillations which are caused by the coherent back-scattering have an amplitude which is independent of $N$. (From ref. 12)
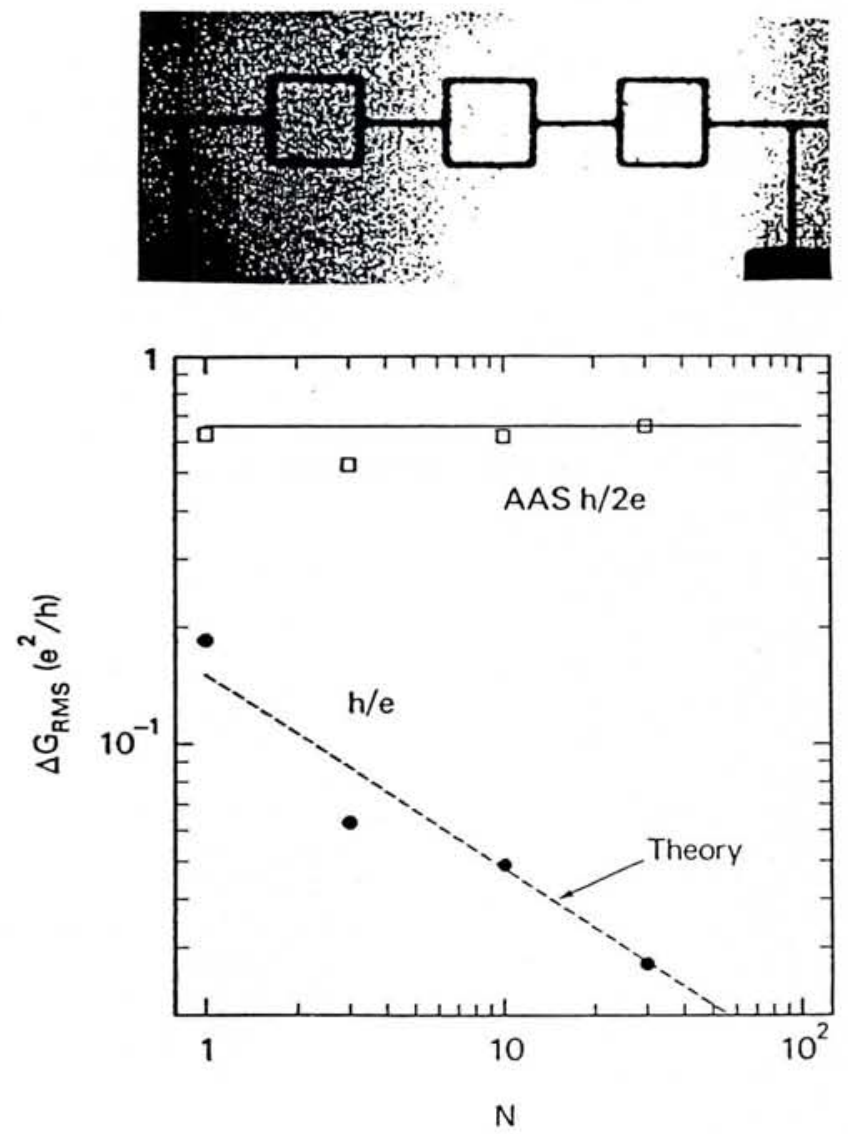
phase coherence length $L_{\varphi}$. Since the magnetoresistance has been measured at high fields, the AAS $h / 2 e$ oscillations are not present. The peak appearing at low frequencies results from the slowly varying aperiodic background.

\section{Transition from Mesoscopic towards Macroscopic Behaviour}

When the number $N$ of mesoscopic units in a sample is not very large, the transition towards the macroscopic regime will be incomplete. This is a direct consequence of the slow, statistical character of the impurity averaging. This slow averaging has been beautifully illustrated by experiments with small arrays of $\mathrm{Ag}$ loops [12]. These arrays were obtained by a combination of electron-beam lithography in a scanning electron microscope and lift-off techniques. In Fig. 4 an electron micrograph for a typical sample with $N=3$ is shown. The loop structure consists of line segments with a width $w \cong 70 \mathrm{~nm}$, a thickness $d \cong 20 \mathrm{~nm}$ and a length $S \cong$ $0.95 \mu \mathrm{m}$. For the $\mathrm{Ag}$ material, $L_{\omega}$ is limited to a value of about $2 \mu \mathrm{m}$ at low temperatures. The loops can therefore be considered as independent mesoscopic units since only interference effects within a single loop will be important. Fig. 4 also shows the variation of the $h / 2 e$ (AAS) and the $h / e$ oscillation amplitude for different $N$. Since the AAS oscillations do not depend upon the amount of impurity averaging, their amplitude is independent of $N$. On the other hand, the $h / e$ oscillation amplitude varies inversely proportional to $\sqrt{ } N$, confirming the stochastic character of the impurity averaging.

In order to estimate the reduction of the $h / e$ effect for the cylinder geometry (see Fig. 2), we can chop the macroscopic cylinder with height $H \cong 1 \mathrm{~cm}$ into $N$ $\cong 10^{6}$ mesoscopic loops with thickness $d \sim 10 \mathrm{~nm}$. The impurity averaging which occurs on a macroscopic scale, will reduce the $h / e$ oscillation amplitude by a factor $\sqrt{ } N \cong 10^{3}$. Since for the cylinder geometry, the $h / 2 e$ oscillations can barely be resolved from the intrinsic noise produced by the cylinder resistance, the $h / e$ oscillations will remain invisible.

The equivalence between the cylinder geometry and a large array of mesoscopic loops has also been confirmed experimentally [13]. The magnetoresistance for an array and a cylinder looks identical. At low fields, the $h / 2 e$ oscillations dominate. At higher fields, the oscillations are damped and replaced by a slowly varying background, which is caused by the penetration of the $\vec{B}$ field
Fig. 5 - Four-terminal measurement of the magnetoconductance of a mesoscopic Au line with and without a loop attached to the outside of the contact probes. The two lines have a length of $2 \mu \mathrm{m}$ and a linewidth of $62 \mathrm{~nm}$. The attached loop has a diameter of $0.7 \mu \mathrm{m}$. The line without the loop (left) shows only aperiodic fluctuations. On the other hand, the line with the loop (right) shows additional oscillations with flux period h/e. This Aharonov-Bohm experiment clearly confirms the non-local nature of the interference effects in mesoscopic samples. (From ref. 16)
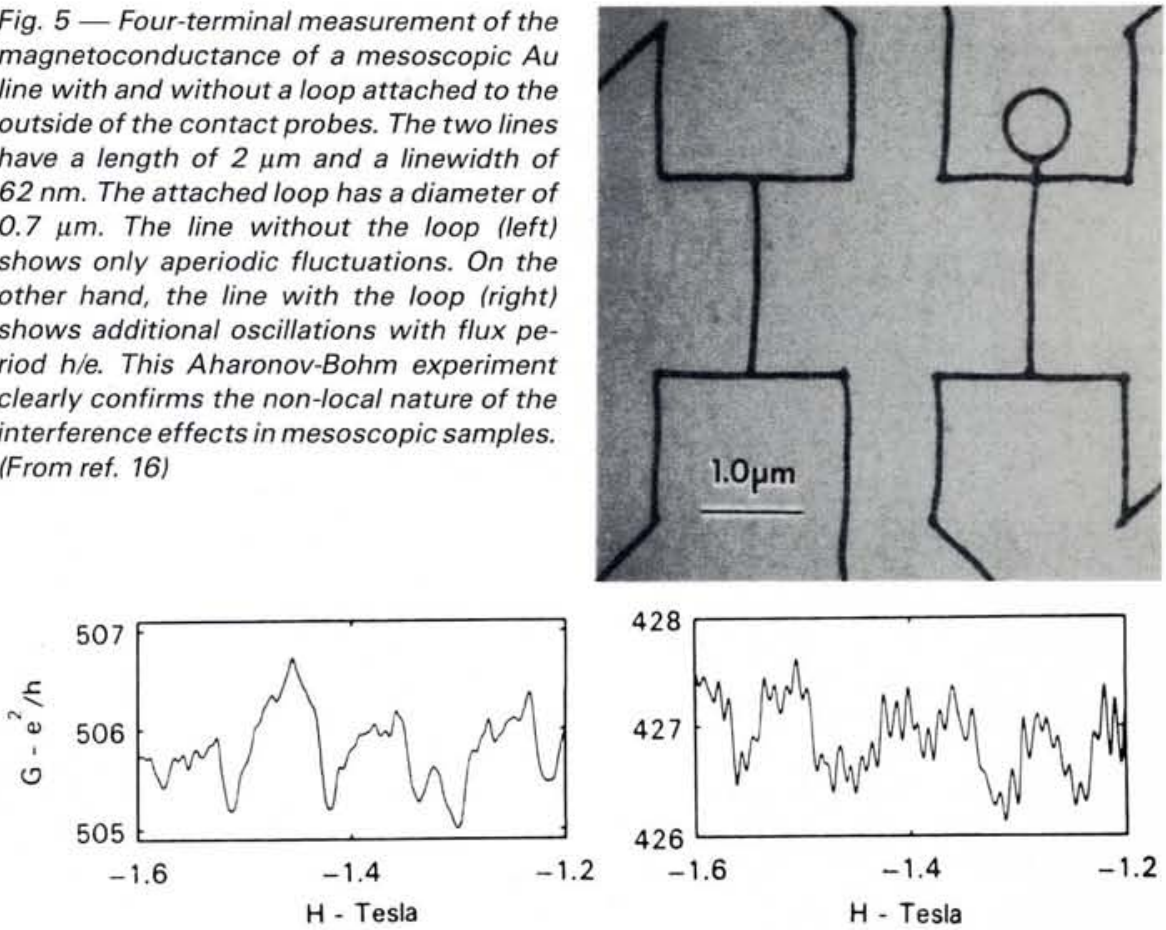

into the arms of the loop or into the cylinder wall.

\section{Looking to the Future}

The presence of the solid-state Aharonov-Bohm effect in mesoscopic structures clearly demonstrates that the conduction electrons in a metal have a welldefined wave character. Similar experiments on semiconductor inversion layers [14] which have not been discussed in this review, confirm that the wave character of the charge carriers also influences the conduction process in mesoscopic doped semiconductors. As indicated by Büttiker [15], the presence of the interference effects on a scale $L_{\text {o }}$ inevitably implies the non-local nature of the flux quantization effects. The electronic properties of mesoscopic samples will therefore also be influenced by regions outside the voltage probes (see Fig. 1).

Umbach, Santhanam, Webb and one of the authors [16], have recently provided a very graphic and easy to understand demonstration of the non-local interference effects.

The results presented in Fig. 5 imply that a four-terminal measurement fails in the mesoscopic regime. Consequently, the classical "scaling down" approach to modeling small circuits is reaching its limits. The sensitivity of the interference to the specific impurity configuration will result in an important electronic noise at higher temperatures when defects can move in a metal or charge traps are present in a semiconducting device. However, when the periodic Aharonov-Bohm oscillations can be made large enough, it is also conceivable that devices based on the quantum interference will become important in the future.

\section{REFERENCES}

[1] Aharonov Y. and Bohm D., Phys. Rev. 115 (1959) 485.

[2] Landauer R., unpublished IBM proposal. [3] Landauer R. in Localization, Interaction berg) 1985 , p. 38.

[4] Gefen Y., Imry Y. and Azbel M.Ya., Phys. Rev. Lett. 52 (1984) 129.

[5] Lee P.A., Sone A.D. and Fukuyama H., Phys. Rev. B 35 (1987) 1039.

[6] Bergmann G., Phys. Rep. 107 (1984) 1.

[7] Altshulez B.L., Aronov A.G. and Spivak B.Z., JETP Lett. 33 (1981) 94.

[8] Gijs M., Van Haesendock C. and Bruynseraede Y., Phys. Rev. Lett. 52 (1984) 2069. [9] Sharvin D.Yu. and Sharvin Yu.V., JETP Lett. 34 (1981) 272.

[10] Webb R.A., Washburn S., Umbach C.P. and Laibowitz R.B., Phys. Rev. Lett. 54 (1985) 2696.

[11] Stone A.D., Phys. Rev. Lett. 54 (1985) 2692.

[12] Umbach C.P., Van Haesendonck C., Laibowitz R.B., Washburn S. and Webb R.A., Phys. Rev. Lett. 56 (1986) 386. Gandit P., Phys. Rev. Lett. 53 (1984) 718; Dolan G.J., Licini J.C. and Bishop D.J., Phys. Rev. Lett. 56 (1986) 1493.

[14] Datta S. et al., Phys. Rev. Lett. 55 (1985) 2344; Skocpol W.J. et al., Phys. Rev. Lett. 56 (1986) 2865.

[15] Büttiker M., Phys. Rev. B 32 (1985) 1846.

[16] Umbach C.P., Santhanam P., Van Haesendonck C. and Webb R.A., Appl. Phys. Lett. 50 (1987) 1289. and Transport Phenomena (Springer, Heidel-

[13] Pannetier B., Chaussy J., Rammal R. and 